\title{
A Gesture Oriented Android Multi Touch Interaction Scheme of Car
}

\author{
Feilong Xu \\ ${ }^{1}$ Institute of Information Technology, Jiangxi University of Technology, Nanchang 330098, China
}

Keywords: Multi touch; Car and interaction; Mobile terminal; Android platform

\begin{abstract}
. with the development of computer technology, more and more operation command, the function is more and more strong, the future of the human vehicle interaction have higher requirements. Whether it has convenience! Concise! The appearance of the car and interaction is a key technology which restric ts the development of the "mobile terminal car and interaction of human and vehicle driving to provide communication interface, design of its direct impact on the share of the smart car sales market". This paper focuses on the analysis of the development and characteristics of mobile terminal car and interaction, and then this paper studies the human vehicle interface and interactive mode of Android platform, and tested with Android intelligent for the definition and identification of the whole interaction gesture platform, verified the interaction method of reliability, availability and scalability.
\end{abstract}

\section{Introduction}

Since the birth of the computer, in the development process shrinking volume, computing power will continue to improve, and eventually lead to the popularity of computer "Meanwhile, with the progress of network and mobile communications technology, the calculation showing trends freedom and flexibility and gradually move toward pervasive computing mode "network structure of traditional distributed systems put this dispersion is further extended to a wider geographical area, but for computing resources can be shared by multiple users use the Internet together is still not very facilitate "the emergence of LAN and workstation sharing and use of resources makes it easier," with the development of mobile computing, wireless networks and the emergence of the make computing more freely, the user can be anywhere in the coverage of a wireless communications net work system! Any time access to system resources, emerging pervasive computing model will calculate a more liberalized, anyone can at any time! All places anywhere to get the required computing services, computing will be everywhere, filled in need of services to achieve and interactive computing resources, "the user through the user interface, multimedia user interface appears before the user interface has been from the text interface, and graphical interface in two stages, "the text interface, the user can only be carried out by text commands and computer interaction, feedback obtained is presented in the form of text," graphical user interface, the interface only two media: text and graphics (image) , are static media. "

The current human-computer interaction is mainly a general purpose computer graphical user interface with the application of in-depth, the drawbacks of this approach have become increasingly prominent, people then proposed natural computer interaction technology. Human-centered! Nature! Efficiency will be the main goal of the development of a new generation of human-computer interaction. 


\section{Android platform, human-computer interaction}

As shown in Fig. 1, based on the multi-touch gesture interaction mainly include natural to define the natural gestures, the touch signal acquisition, gesture recognition of three parts.

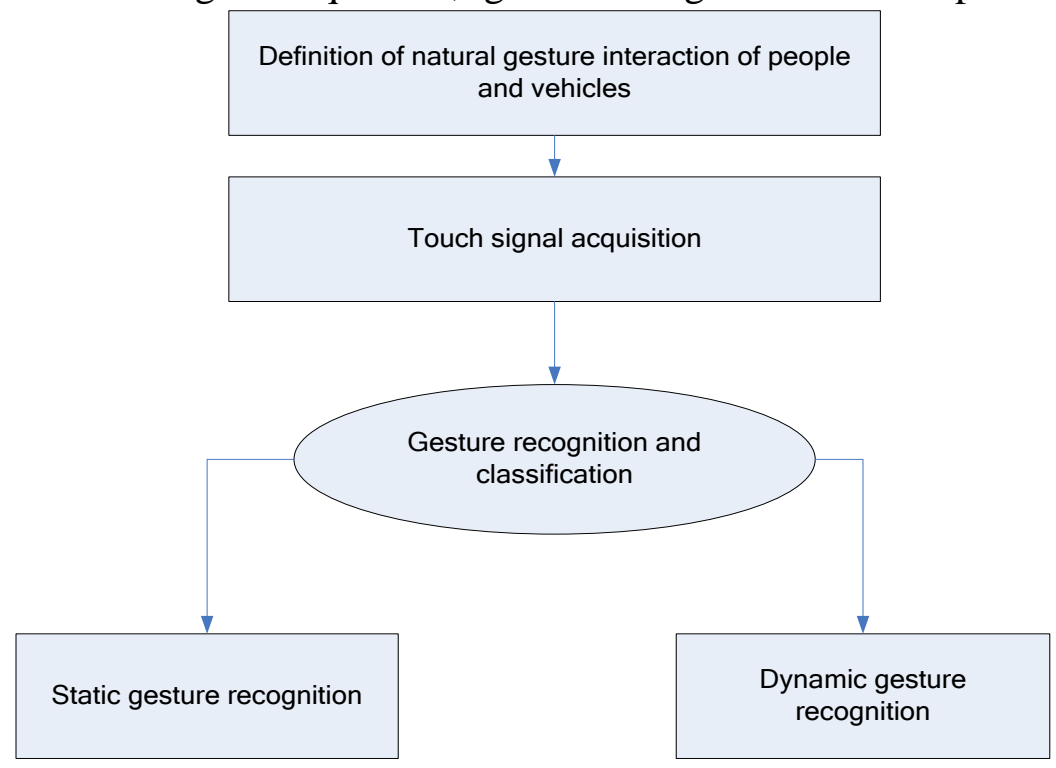

Fig. 1 natural interaction gestures implementation structure

Natural interaction gestures set definition. According to the command post job tasks and interactive relationship between the characteristics of the contact position manpower naturally open when the plane touched, the definition of static and dynamic types of touch gestures, gestures to establish a collection of databases, including a collection of static and dynamic touch gestures touch gestures collection. Static single finger touch gestures collection includes static gestures, finger static gestures, three-finger static gestures, four-finger gestures and fingers static static gestures. Each static finger movements and gestures operational semantics, as shown in Table 1. In order to enrich the interaction semantics interactive gesture set, for the same contact number of interactive gestures but also define different specific operations gestures. Fig. 2 shows a subset of the static four-finger gesture static gestures, static gestures guaranteed four fingers on the basis of the recognition rate can be divided into four fingers of one hand and the four fingers of both hands static gestures. For ease of description, the various gestures using $\mathrm{L}, \mathrm{R}$, and $\mathrm{A}, \mathrm{B}, \mathrm{C}, \mathrm{D}, \mathrm{E}$ to express a combination of letters of the alphabet, wherein $\mathrm{L}, \mathrm{R}$ represent the left and right, $\mathrm{A}, \mathrm{B}, \mathrm{C}, \mathrm{D}, \mathrm{E}$, respectively, represent a hand thumb, index finger, middle finger, ring finger and little finger. 


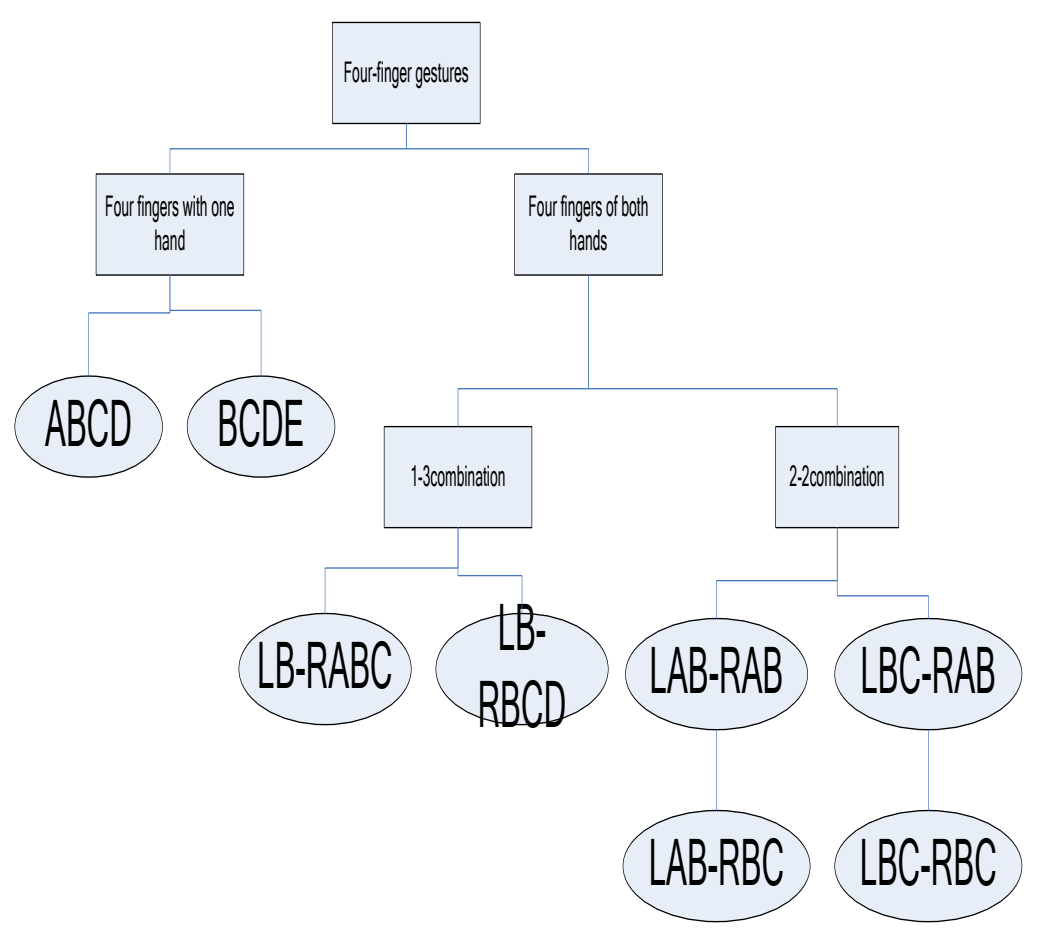

Fig. 2 four-finger gestures collection

Dynamic touch gestures including pan gesture set, arcs shift gestures, hands rotate gestures, one hand rotation gestures, zoom gestures, pinch gestures and take extracts gesture release, including panning gestures, arc shift gestures, pinch gestures and take extracts release defaults to a single gesture hand gestures, zoom gesture default gesture with both hands, rotate gesture to distinguish one hand and hands rotate gestures.

Design methods need to identify the characteristics of the set of gesture-based interactive gestures as defined above, so as to ensure a high recognition rate and the recognition efficiency. Fig. 3 is a static gesture recognition process. For static gestures, the staff naturally open when touched, because of the distance and location of different finger length, contacts between any two contacts constituted differently and each vertex of the angle of the polygon also presents certain characteristics. When individuals through different height planes touching fingers naturally open contact distance measurement data statistics, determine the maximum distance between contacts with one hand, as a basis for the left and right contacts into two sets.

The first touch point in the left of the screen as a reference point, followed by calculation of the distance from the reference point between the rest of the respective touch points when the distance is less than or equal to the threshold value t1 (the operator based on the palm of one hand in a comfortable, non-tension stretching sense finger maximum distance data flared statistics to determine the threshold $\mathrm{t}$, the range of $18 \sim 21 \mathrm{~cm}$ ), it is determined that this touch point is valid; when the distance is larger than the threshold value $t 1$, determine if this touch point the interference point, and the collected from the touch point $\mathrm{M}$ deleted.

\section{Identification and testing}

Use 65 infrared multi-touch screen as an experimental device, the definition of four-finger gestures, hands rotate gestures, zoom gesture with both hands, arcs shift gestures, pinch take gestures, three-finger gestures to extract 500 times recognition test, the results are shown in Table 1 (in which four data refer to the different gestures in the gesture recognition results taking the mean). 
Table 1 Results

\begin{tabular}{|c|c|c|c|c|c|c|}
\hline Gesture & $\begin{array}{c}\text { Four } \\
\text { fingers }\end{array}$ & $\begin{array}{c}\text { Hands } \\
\text { Zoom }\end{array}$ & Arc shift & Take pinch & Take pinch & $\begin{array}{c}\text { Hands } \\
\text { rotation }\end{array}$ \\
\hline $\begin{array}{c}\text { Average time } \\
(\mathrm{s})\end{array}$ & 0.026 & 0.893 & 0.533 & 0.663 & 0.623 & 0.967 \\
\hline Correct rate & $99.96 \%$ & $99.87 \%$ & $99.74 \%$ & $99.78 \%$ & $99.80 \%$ & $99.87 \%$ \\
\hline
\end{tabular}

\section{Conclusions}

The data can still be seen from the above definition and gesture recognition method described herein has the following advantages:

Identification accuracy. Based on a job application definition touch gesture command post, according to the command post operating characteristics distinguish static gestures and dynamic gestures, gestures collection of well-defined, and the data set based on anatomy and physiology and movement distance threshold characteristic parameters are static gestures and dynamic gesture recognition, parameters required for simple and clear identification features. Therefore, the identification method can achieve high recognition accuracy.

Fast the recognition speed. Compared to traditional methods, this method is not complicated signal processing, resource and time consuming is relatively small, relatively stable combined command post operational semantics, identify the required parameters of simple and clear, and therefore a shorter response time gesture recognition.

Scalability. This method requires less characteristic parameters, and hardware coupling does not close, touch-based signal acquisition, screen position coordinates of the touch point of the record, for a variety of different optical sensors multi-touch screen device, so the versatility and scalability good low cost.

\section{Acknowledgements}

This work was supported by Project of Jiangxi University of Technology [No. xtcx201320]

\section{References}

[1] SHU Xian-hua, DU Zhen-jun, CHEN Rong. Research onMobile Location ServiceDesi gn based on Android. Pro-ceedings of theIEEE Conf WICOM09 . 2009

[2] Hu JF, Mu ZD. Smart Car Security System by Using Biometrics Based on EEG [J]. 2013 2nd International Conference on Frontiers of Energy and Environment Engineering, ICFEEE 2013: 1047-1049.

[3] Lee BG, Chung WY. A Smartphone-Based Driver Safety Monitoring System Using Data Fusion [J]. Sensors, 2012, 17536-17552.

[4] Saini V, Saini R. Driver Drowsiness Detection System and Techniques: A Review [J]. International Journal of Computer Science and Information Technologies, 2014, 4245-4249.

[5] Vasavi D, Krishnaiah RV. Online detection of drowsiness using brain and visual information [J]. International Journal of Instrumentation Electrical Electronics Engineering, 2013, 31-34. 
[6] Fuletra JD, Bosamiya D. A Survey on Driver's Drowsiness Detection Techniques [J]. International Journal on Recent and Innovation Trends in Computing and Communication, 2013, 816-819.

[7] ZHANU Guo-wu. intelligent transportation systems and the integrated transport information service[J].Journal of Transportation Systems Engineering and Information Technology, 2013, 13(2):1-8.(in Chinese).

[8] Viktor M S, Kenneth C. Big data[M].Hangzhou:Zhejiang People' s Publishing House, 2013.(in Chinese).

[9] ZHOU Xiao-fang,LU Jia-heng,Li Cui-ping, et al.Big data challenges from the perspective of datament[J].Communication of China Computer2012, 8(9):16-21.(in Chinese).

[10] Ekram H, Garland C,Victor C M L, et al. Vehicular telematics over heterogeneous wireless networks: a survey[J].Computer Communications, 2012, 33(7): 775-793. 